\title{
Stability of a Non-Linear Exponential Autoregressive Model
}

\author{
Abdulghafoor Jasem Salem, Abeer Abdulkhaleq Ahmad \\ Department of Mathematics, College of Computer Sciences and Mathematics, University of Mosul, Mosul, Iraq \\ Email: abeerdabagh1@gmail.com
}

How to cite this paper: Salem, A.J. and Ahmad, A.A. (2018) Stability of a Non-Linear Exponential Autoregressive Model. Open Access Library Journal, 5: e4482. https://doi.org/10.4236/oalib.1104482

Received: March 12, 2018

Accepted: April 21, 2018

Published: April 24, 2018

Copyright $\odot 2018$ by authors and Open Access Library Inc.

This work is licensed under the Creative Commons Attribution International License (CC BY 4.0).

http://creativecommons.org/licenses/by/4.0/

\begin{abstract}
In this paper, a non-linear exponential autoregressive model has been studied by applying the local linearization technique. The obtained results have been implemented in a time series which represents number of diabetic patients for ten years period.
\end{abstract}

\section{Subject Areas}

Mathematical Statistics

\section{Keywords}

Time Series, Local Linearization Technique, Akaike's Information Criteria

\section{Introduction}

Time series are a record of values for any volatile quantity measured by different points of time, for example, measuring daily temperatures, measuring the voltage of the electric circuit for each single dish, measuring price indices for each month ...

The time series are usually based on basic features such as stability, linearity, normality, and how to handle non-prophetic time series to construct appropriate mathematical models. Our research will focus on the study of the stability of a nonlinear model (first-order polynomial) because this model usually has a nonlinear behavior so the linear approximation method suggested by the researcher Uzaki was used to study the characteristics of the model, (Note source [1]).

\section{Time Series}

\subsection{Time Series}

Is a set of observations that are generated continuously over time? Any time se- 
ries is characterized by its temporal order and successive observations are usually independent of each other. The conjugation process is defined as a family of random variables $\left\{x_{t}, t \in T\right\}$ where $t$ is the index $(t \in T)$ and $T$ is a set index and if $t$ is the time, it is called $\left\{x_{t}, t \in T\right\}$.

\subsection{Specifications Model Selection Criteria [2] [3]}

In the time series analysis and more generally in data analysis, there are appropriate models that can be used to represent the studied data. Sometimes the selection is easy, but sometimes the choice is based on the self-binding function (ACF) (PACF) or error box rate (MSE). Therefore, other criteria are used to facilitate the process of testing the best model suitable for the studied series.

\section{Akaike's Information Criteria of Nonlinear Time Series Model [4]}

The AACI standard, abbreviated to AIC, is one of the general criteria used to select the best model among a range of models. The model that reduces the Akayki standard is the best model. This criterion is also used to determine the best ranking of the model from the family of models of different classes. The standard of Acaique which is commonly used in linear time series models is:

$$
\operatorname{AIC}(p)=(N-P) * \ln \left(\sigma_{z}^{2}\right)+2 * m
$$

This standard may have more than one small value depending on the assumption that the data to be modeled follows the normal distribution. In applications, we look for a model that is highly congruent with reality, i.e., the model in which residual values are normal distribution with the smallest possible variance, which is denoted here by $\sigma_{z}^{2}$.

The general formulation of the AIC for time series models, namely nonlinear self-regression models, is:

$$
\operatorname{AIC}(P)=(N-P) * \ln \left(\sigma_{z}^{2}\right)+2 * m
$$

$N$ is the number of observed data, $P$ is the order of the model $\sigma^{2}$ is the estimated value of the variance of the model locks, and $\mathrm{m}$ is the number of parameters in the model.

\subsection{Criteria of (BIC)}

It is a development of the AIC standard developed by the world Akaike, called the Bayesian Information Criteria and the abbreviation BIC $(P)$ and is defined as:

$$
\operatorname{BIC}(P)=n \ln \left(\sigma_{z}^{2}\right)+m \ln (n)
$$

where $N$ is the number of parameters, $m$ the number of parameters, and $\sigma^{2}$, the variance of residues. The standard formula for this standard (Normalized BIC) is then taken by dividing BIC by $n$ and denoted by the symbol NBIC,

$$
\mathrm{NBIC}=\frac{\mathrm{BIC}}{n}
$$


It is preferable to use the BIC criterion on the AIC because the latter has a large number of parameters and does not take into account the issue of model stability.

\subsection{Box-Cox Transformation [5]}

Let $\left\{X_{t}\right\}$ represent a time series that includes $n$ views $x_{n}, \cdots, x_{2}, x_{1}$. Box-Cox conversion defines the following:

$$
X_{t}^{(\lambda)}= \begin{cases}\lambda^{-1}\left[\left(X_{t}+c\right)^{\lambda}\right] ; & \lambda \neq 0 \\ \log \left(X_{t}+c\right) ; & \lambda=0\end{cases}
$$

And $c$ is a fixed amount that usually takes a large enough value to make the observations positive and $0 \leq \lambda \leq 1$.

\subsection{Exponential Autoregressive Model [6]}

Oda and Ozaki in 1977 defined the exponential self-regression model of rank $p$ as follows:

$$
X_{t}=\sum_{i=1}^{p}\left(\alpha_{i}+\pi_{i} \mathrm{e}^{-x_{t-i}^{2}}\right) x_{t-i}+Z_{t}, i=1,2,3, \cdots, p
$$

The $z(t)$ white noise (white noise) follows the natural distribution at zero and the variation $\sigma_{z}^{2}$ and $i$, ii $\alpha$ constant quantities (model parameters).

\subsection{The First-Order Exponential Regression Model (Proposed Model)}

Let the time series $\left\{X_{t}\right\}$ where $t= \pm 1, \pm 2, \pm 3, \cdots$

The exponential self-regression model is known as the first rank in the form:

$$
X_{t}=\left(\varnothing_{1}+\pi_{1} \mathrm{e}^{-x_{t-1}^{2}}\right)^{p} x_{t-1}+Z_{t}, \quad p=2,3, \cdots
$$

where $\left\{Z_{t}\right\}$ is white noise, a, $\varnothing_{1}$ and $\pi_{1}$ are constant quantities.

The Oda and Ozaki models have shown that the end cycle of model 5 can be determined by the following proof.

\section{A Theorem}

Let the time series $\left\{X_{t}\right\}$ represent the model of exponential self-regression from the first rank:

$$
X_{t}=\left(\alpha_{1}+\pi_{1} \mathrm{e}^{-x_{t-1}^{2}}\right) x_{t-1}+Z_{t}
$$

The end course of the course $X_{t+1}, X_{t+2}, \cdots, X_{t+q}, q$ For the model to be orbitally stable if the condition is met:

$$
\left|\prod_{j=1}^{q}\left[\alpha_{j}+\pi_{j}\left(1-2 x_{t+q-j}^{2}\right) \mathrm{e}^{-x_{t+q-j}^{2}}\right]\right|<1
$$

The proof: note that [4]. 


\subsection{Case}

Let us have the model

$$
X_{t}=\left(\varnothing_{1}+\pi_{1} \mathrm{e}^{-x_{t-1}^{2}}\right) x_{t-1}+Z_{t}
$$

The end cycle of the cycle (if any) of the above model is orbital stable.

If the condition is satisfy:

$$
\left|\frac{\xi_{t+q}}{\xi_{t}}\right|<1
$$

The proof: note that [7].

\section{2. (Singular Point): [7]}

A single point is defined as the point that satisfies the following condition: any path of the model starts from a point close enough to it when $t \rightarrow \infty$ or $t \rightarrow-\infty$.

If the path is close to where $t \rightarrow \infty$ فأن , the single point is stability and vice versa if the path is close to when $t \rightarrow-\infty$ فأن, the single point is not propelled.

\section{3. (Limit Cycle) [7]}

The end cycle of the form is defined as the closed and isolated path $x_{t}=\left(x_{t}, x_{t+1}, x_{t+2}, \cdots, x_{t+q}\right)$ where $q$ is a positive integer. The track is closed because if you are the primary values $\left(x_{t}, x_{t+1}, x_{t+2}, \cdots, x_{t+q}\right)$ belong to the end cycle $\left(x_{1}, x_{2}, x_{3}, \cdots, x_{p}\right)=\left(x_{1+k q}, x_{2+k q}, x_{3+k q}, \cdots, x_{p+k q}\right)$.

For any integer $k$.

Isolated is that any path that starts close to the end cycle sufficiently converges towards the end cycle. When $t \rightarrow \infty$ i or $t \rightarrow-\infty$, if the path is nearing the end cycle when $t \rightarrow$ فأن, the end cycle is a stability and vice versa if the path nearing the end cycle when $t \rightarrow-$ فأن, the end cycle is non-fanatic.

\section{Stability of the Proposed Model}

In this section we will study the stability of model (5) using the method of approximation of the local line.

Let us have the model defined by Equation (5):

$$
X_{t}=\left(\varnothing_{1}+\pi_{1} \mathrm{e}^{-x_{t-1}^{2}}\right)^{p} x_{t-1}+Z_{t}, \quad p=1,2, \cdots
$$

The first When $p=1$ and from case note (10) we find that:

- Single point:

A single point shall be present if the following condition is met:

$$
\Rightarrow \xi= \pm \sqrt{-\ln \left(\frac{1-\varnothing_{1}}{\pi_{1}}\right)}
$$

- Single point stability:

$$
\Rightarrow \xi_{t}=\left(1+2\left(1-\varnothing_{1}\right) \ln \left(\frac{1-\varnothing_{1}}{\pi_{1}}\right)\right) \xi_{t-1}
$$


The last equation represents a linear self-regression model of the first order and its stability condition is to be

$$
|\mu|=\left|1+2\left(1-\varnothing_{1}\right) \ln \left(\frac{1-\varnothing_{1}}{\pi_{1}}\right)\right|<1
$$

where $\mu$ is the root of the characteristic equation of the model above.

- Stability of the end cycle:

$$
\Rightarrow\left|\frac{\xi_{t+q}}{\xi_{t}}\right|=\left|\prod_{i=1}^{q}\left(\varnothing_{1}+\pi_{1} \mathrm{e}^{-\left(X_{t+q-i}\right)^{2}}\left(1-2\left(X_{t+q-i}\right)^{2}\right)\right)\right|<1
$$

Second: Let $p=2$ in the proposed model (5) we get

$$
X_{t}=\left(\varnothing_{1}+\pi_{1} \mathrm{e}^{-x_{t-1}^{2}}\right)^{2} x_{t-1}+Z_{t}
$$

- Single point:

Using the definition of single point and compensation in the above model we get

$$
\begin{gathered}
\xi=\left(\varnothing_{1}+\pi_{1} \mathrm{e}^{-\xi^{2}}\right)^{2} \xi \\
\xi=0 \\
\xi \neq 0
\end{gathered}
$$

Zero point, and to find non-zero individual points assume that $\xi \neq 0$.

After performing the easy sports we get

$$
\xi= \pm \sqrt{-\ln \left(\frac{-\varnothing_{1} \pm \sqrt{2 \varnothing_{1}^{2}-1}}{\pi_{1}}\right)}
$$

- Single point stability:

Using hypotheses similar to those used for $p=1$, we obtain:

We get the equation:

$$
\xi_{t}=A \xi_{t-1}
$$

where

$$
\begin{aligned}
A= & \varnothing_{1}^{2}+2 \varnothing_{1} \pi_{1} \frac{-2 \varnothing_{1} \pi_{1} \pm \sqrt{\left(2 \varnothing_{1} \pi_{1}\right)^{2}-4\left(\pi_{1}^{2}\right)\left(1-\varnothing_{1}^{2}\right)}}{2 \pi_{1}^{2}} \\
& +\pi_{1}^{2} \frac{-2 \varnothing_{1} \pi_{1} \pm \sqrt{\left(2 \varnothing_{1} \pi_{1}\right)^{2}-4\left(\pi_{1}^{2}\right)\left(1-\varnothing_{1}^{2}\right)}}{2 * \pi_{1}^{2}} \\
& -4 \varnothing_{1} \pi_{1} \frac{-2 \varnothing_{1} \pi_{1} \pm \sqrt{\left(2 \varnothing_{1} \pi_{1}\right)^{2}-4\left(\pi_{1}^{2}\right)\left(1-\varnothing_{1}^{2}\right)}}{2 \pi_{1}^{2}} *-\ln \left(\frac{-\varnothing_{1} \pm \sqrt{2 \varnothing_{1}^{2}-1}}{\pi_{1}}\right) \\
& -4 \pi_{1}^{2} * \frac{-2 \varnothing_{1} \pi_{1} \pm \sqrt{\left(2 \varnothing_{1} \pi_{1}\right)^{2}-4\left(\pi_{1}^{2}\right)\left(1-\varnothing_{1}^{2}\right)}}{2 \pi_{1}^{2}} *-\ln \left(\frac{-\varnothing_{1} \pm \sqrt{2 \varnothing_{1}^{2}-1}}{\pi_{1}}\right)
\end{aligned}
$$

Equation (10) represents a first-order differential equation and the stability 
condition is that the root of the characteristic equation is less than one

$$
|A|<1
$$

- Stability of the end cycle: using case (10) and assuming that $X_{s}=X_{s}+\xi_{s}$.

We get

$$
X_{t}+\xi_{t}=\left(\varnothing_{1}+\pi_{1} \mathrm{e}^{-\left(X_{t-1}+\xi_{t-1}\right)^{2}}\right)^{2}\left(X_{t-1}+\xi_{t-1}\right)
$$

Since $\xi_{t}(t)$ is too small we assume that

$$
\xi_{t}^{n} \rightarrow 0 ; \forall n \geq 2
$$

Using similar mathematical processes we get the:

Let $t=t+q$,

$$
\begin{aligned}
\xi_{t+q}= & \left(\varnothing_{1}^{2}-4 \varnothing_{1} \pi_{1} \mathrm{e}^{-X_{t+q-1}^{2}} X_{t+q-1}^{2}+2 \varnothing_{1} \pi_{1} \mathrm{e}^{-X_{t+q-1}^{2}}\left(1-2 X_{t+q-1}^{2}\right)\right. \\
& \left.+\pi_{1}^{2} \mathrm{e}^{-2 X_{t+q-1}}\left(1-4 X_{t+q-1}^{2}\right)\right)\left(\varnothing_{1}^{2}-4 \varnothing_{1} \pi_{1} \mathrm{e}^{-X_{t+q-2}^{2}} X_{t+q-2}^{2}\right. \\
& \left.+2 \varnothing_{1} \pi_{1} \mathrm{e}^{-X_{t+q-2}^{2}}\left(1-2 X_{t+q-2}^{2}\right)+\pi_{1}^{2} \mathrm{e}^{-2 X_{t+q-2}^{2}}\left(1-4 X_{t+q-2}^{2}\right)\right) \\
& * \cdots *\left(\varnothing_{1}^{2}-4 \varnothing_{1} \pi_{1} \mathrm{e}^{-X_{t}^{2}} X_{t}^{2}+2 \varnothing_{1} \pi_{1} \mathrm{e}^{-X_{t}^{2}}\left(1-2 X_{t}^{2}\right)+\pi_{1}^{2} \mathrm{e}^{-2 X_{t}^{2}}\left(1-4 X_{t}^{2}\right)\right) \xi_{t}
\end{aligned}
$$

The end cycle is stable if the following condition is satisfy:

$$
\begin{aligned}
\Rightarrow\left|\frac{\xi_{t+q}}{\xi_{t}}\right|= & \mid \prod_{i=1}^{q}\left(\varnothing_{1}^{2}-4 \varnothing_{1} \pi_{1} \mathrm{e}^{-X_{t+q-i}^{2}} X_{t+q-i}^{2}+2 \varnothing_{1} \pi_{1} \mathrm{e}^{-X_{t+q-i}^{2}}\left(1-2 X_{t+q-i}^{2}\right)\right. \\
& \left.+\pi_{1}^{2} \mathrm{e}^{-2 X_{t+q-i}^{2}}\left(1-4 X_{t+q-i}^{2}\right)\right) \mid<1
\end{aligned}
$$

This is proof.

Third: In the case of $p>2$ that is

$$
X_{t}=\left(\varnothing_{1}+\pi_{1} \mathrm{e}^{-x_{t-1}^{2}}\right)^{p} x_{t-1}+Z_{t} ; p=3,4, \cdots
$$

- Single point:

Using the definition and compensation in the above model, we get

$$
\xi=\left(\varnothing_{1}+\pi_{1} \mathrm{e}^{-\xi^{2}}\right)^{p} \xi ; p=3,4, \cdots
$$

$\xi=0$ is the single zero point, and to find the non-zero individual points, suppose that $\xi=0$ we get the single point non-zero

$$
\xi= \pm \sqrt{-\ln \left(\frac{1-\varnothing_{1}}{\pi_{1}}\right)}
$$

- Single point stability:

$$
\xi+\xi_{t}=\left(\varnothing_{1}+\pi_{1} \mathrm{e}^{-\xi+\xi_{t-1}^{2}}\right)^{p}\left(\xi+\xi_{t-1}\right) ; p=3,4, \cdots
$$

The solution to the equation above is by using algebraic and numerical processes, and we obtain the stability of the non-zero point of the model.

- Stability of the end cycle:

By using the case (4.1) we get the 


$$
X_{t}+\xi_{t}=\left(\varnothing_{1}+\pi_{1} \mathrm{e}^{-\left(X_{t-1}+\xi_{t-1}\right)^{2}}\right)^{p}\left(X_{t-1}+\xi_{t-1}\right) ; p=3,4, \cdots
$$

Using previous hypotheses, similar mathematical operations in the case of $i=$ 1,2 are obtained

$$
\begin{aligned}
\left|\frac{\xi_{t+q}}{\xi_{t}}\right|= & \prod_{i=1}^{q}\left(\left(\begin{array}{l}
p \\
0
\end{array}\right) \varnothing_{1}^{p}+\left(\begin{array}{c}
p \\
1
\end{array}\right) \varnothing_{1}^{p-1} \pi_{1} \mathrm{e}^{-\left(X_{t+q-i}\right)^{2}}\left(1-2\left(X_{t+q-i}\right)^{2}\right)\right. \\
& +\left(\begin{array}{l}
p \\
2
\end{array}\right) \varnothing_{1}^{p-2}\left(\pi_{1} \mathrm{e}^{-\left(X_{t+q-i}\right)^{2}}\right)^{2}\left(1-4\left(X_{t+q-i}\right)^{2}\right) \\
& +\left(\begin{array}{l}
p \\
3
\end{array}\right) \varnothing_{1}^{p-3}\left(\pi_{1} \mathrm{e}^{-\left(X_{t+q-i}\right)^{2}}\right)^{3}\left(1-6\left(X_{t+q-i}\right)^{2}\right) \\
& \left.+\cdots+\left(\begin{array}{l}
p \\
p
\end{array}\right)\left(\pi_{1} \mathrm{e}^{-\left(X_{t+q-i}\right)^{2}}\right)^{n}\left(1-2 p\left(X_{t+q-i}\right)^{2}\right)\right)
\end{aligned}
$$

And from the last equation we get the

$$
\left|\frac{\xi_{t+q}}{\xi_{t}}\right|=\prod_{i=1}^{q}\left(\sum_{j=0}^{p}\left(\begin{array}{l}
p \\
j
\end{array}\right) \varnothing_{1}^{p-j}\left(\pi_{1} \mathrm{e}^{-\left(X_{t+q-i}\right)^{2}}\right)^{j}\left(1-2 j\left(X_{t+q-i}\right)^{2}\right)\right) .
$$

The exponential self-regression model of the first order and the p-class is a model with a non-zero, stable and non-zero point

$$
\xi= \pm \sqrt{-\ln \left(\frac{1-\varnothing_{1}}{\pi_{1}}\right)}
$$

It has an unstable end cycle.

\section{The Applied Side}

This chapter examines the time series of people with diabetes. As each statistic represents the monthly total number of people infected with this disease, where the building of different mathematical models and using the program Statistica obtained several models. Stability of the proposed models representing the studied series was also studied. The data were obtained from Ibn Sina Educational Hospital in the city of Mosul for the period 2012.

The observations of the time series represent the monthly total number of patients with diabetes in the blood for ten years (2003-2012). When looking at the data available from the first month of 2003 to December 2012 and the lowest value is the number of casualties in 52 in September In 2008 and the highest in December, with 185 cases in 2012.

Figure 1 represents the graph of the original series of numbers of people with diabetes.

Figure 1 shows that the unstable time series fluctuates irregularly and the data has a general trend in the chain (increasing), correlated, and large dispersion. We note that more than $5 \%$ of the self-correlation coefficients fall outside the constraint $( \pm 1.965) / \mathrm{nn}$ (Confidence limits) but close to normal distribution. To 


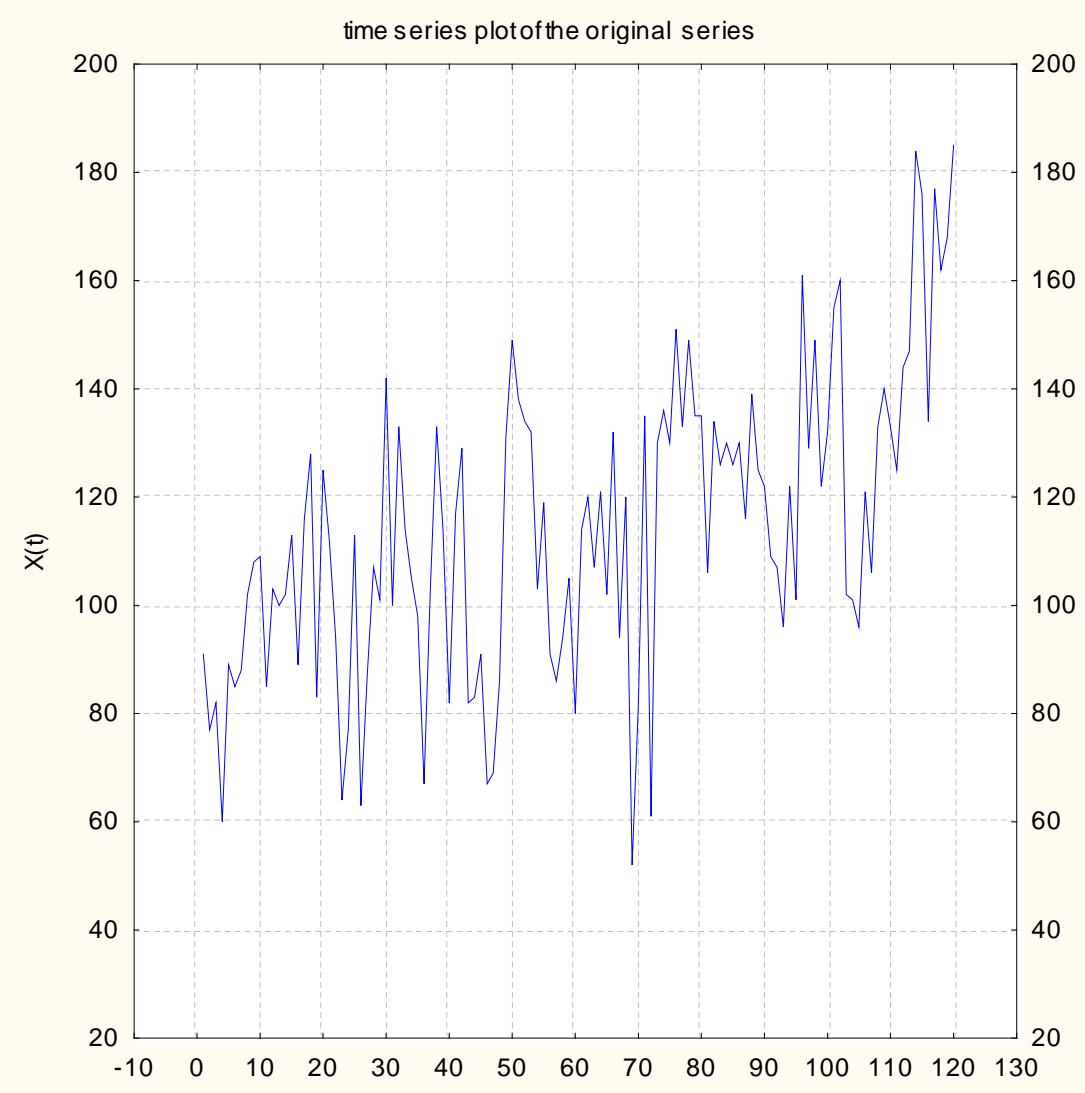

(a)

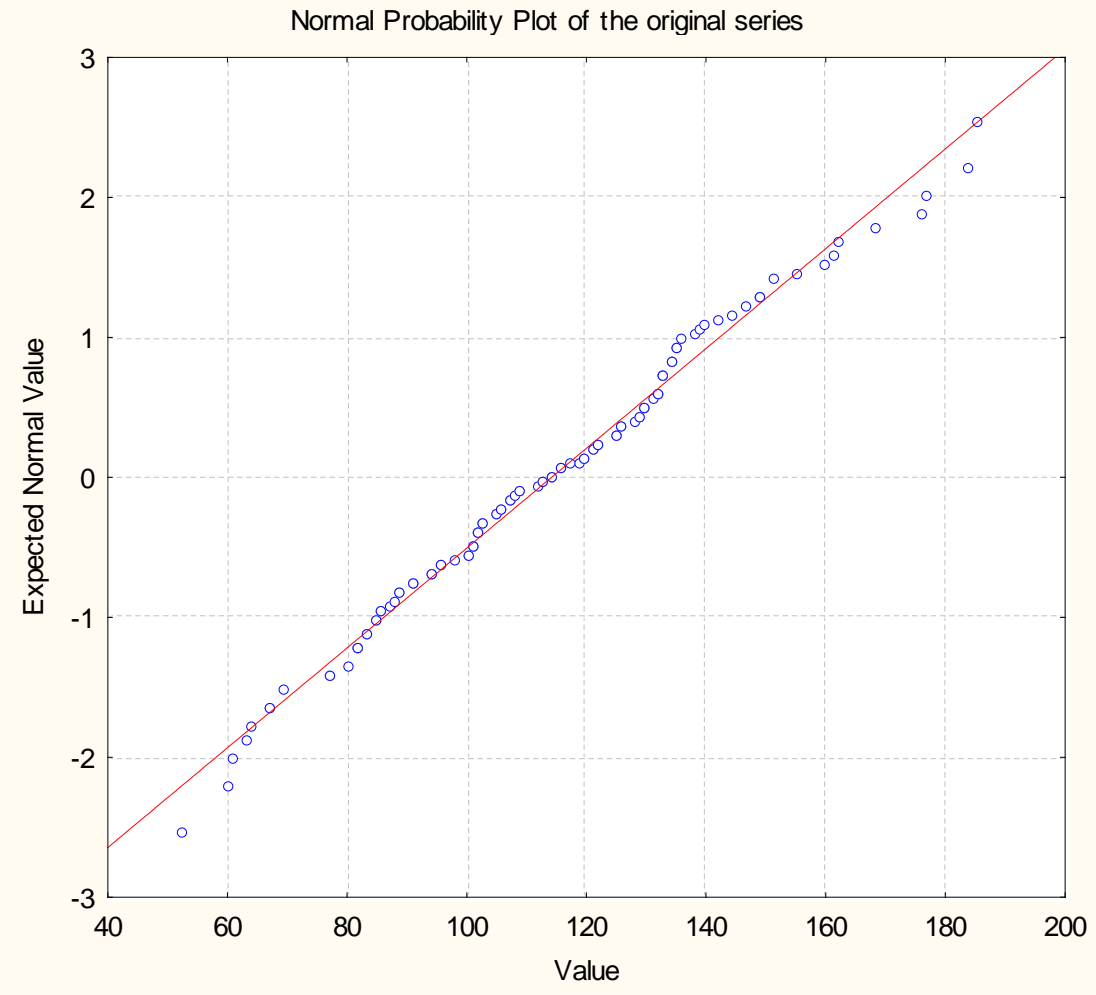

(b) 


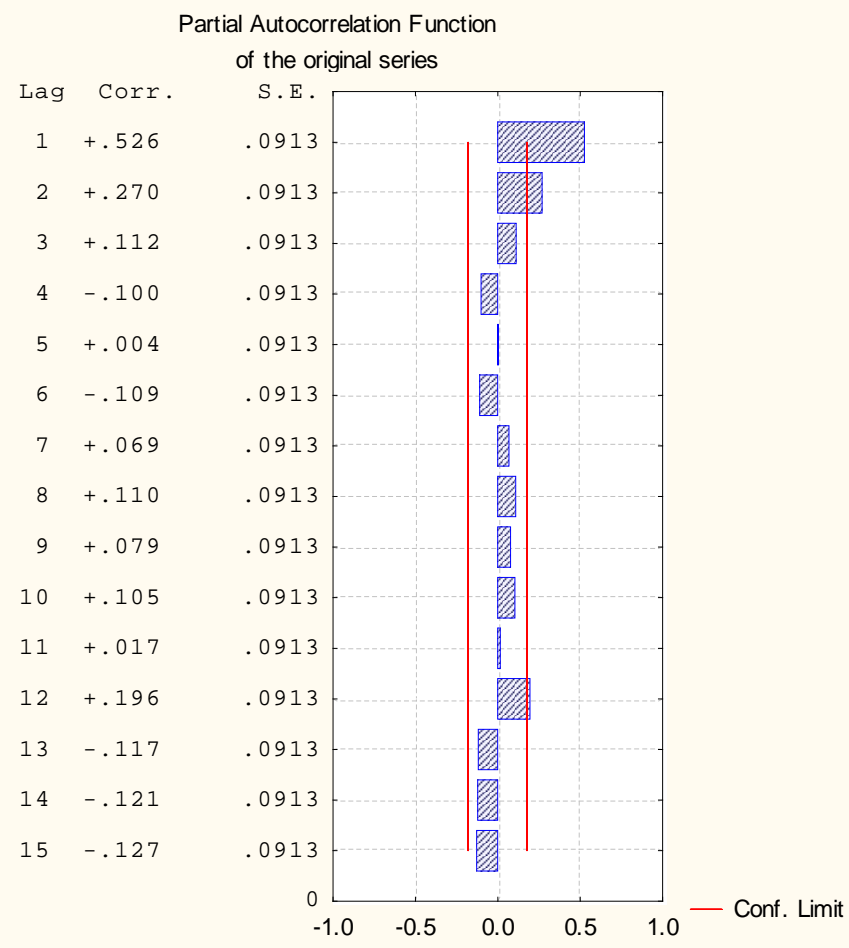

(c)

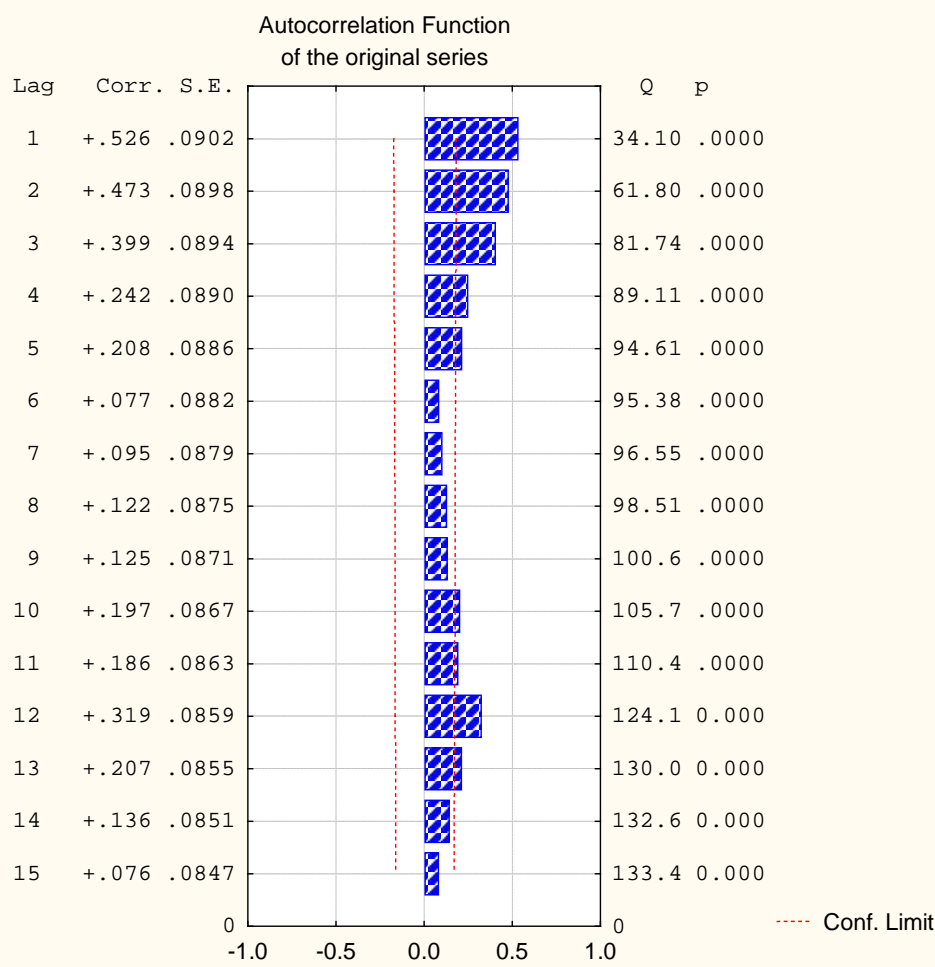

(d)

Figure 1. Represents the graph of the original series of numbers of people with diabetes (a) Represents the graph of the original series of numbers of people with diabetes, (b) Representing the natural drawing and ((c), (d)) Representing the partial self-correlation function and the self-correlation function respectively. 
convert them into stable series we use BOX-COX transformations. The natural logarithm of the data is usually taken (for varying probability), and the differences are then taken to be stable in the mean, i.e.:

$$
y_{t}=\log \left(x_{t}\right)
$$

$x_{t}$ represents the original string, $y_{t}$ represents the converted string, takes the first difference to convert it to a stable in the middle and as follows:

$$
\begin{gathered}
w_{t}=\Delta^{d} y_{t} \\
d=1 ; \Delta=(1-B)
\end{gathered}
$$

where $B$ is the rear displacement motor which is known as:

$$
B^{r} x_{t}=x_{t-r} ; r=1,2, \cdots
$$

Figure 2 represents the graph, the natural graph, the self-correlation function, and the partial auto-correlation function of the converted series.

Figure 2 shows that the chain is stable around the center and correlated with less than $5 \%$ of the self-correlation coefficients outside the constraint $( \pm 1.965) / \mathrm{nn}$ and close to normal distribution.

\section{The Modeling}

In this section we will build mathematical models for the time series of people with diabetes. As we noted from the previous paragraph that the series is stable around the center and oscillates irregularly and periodically, indicating the

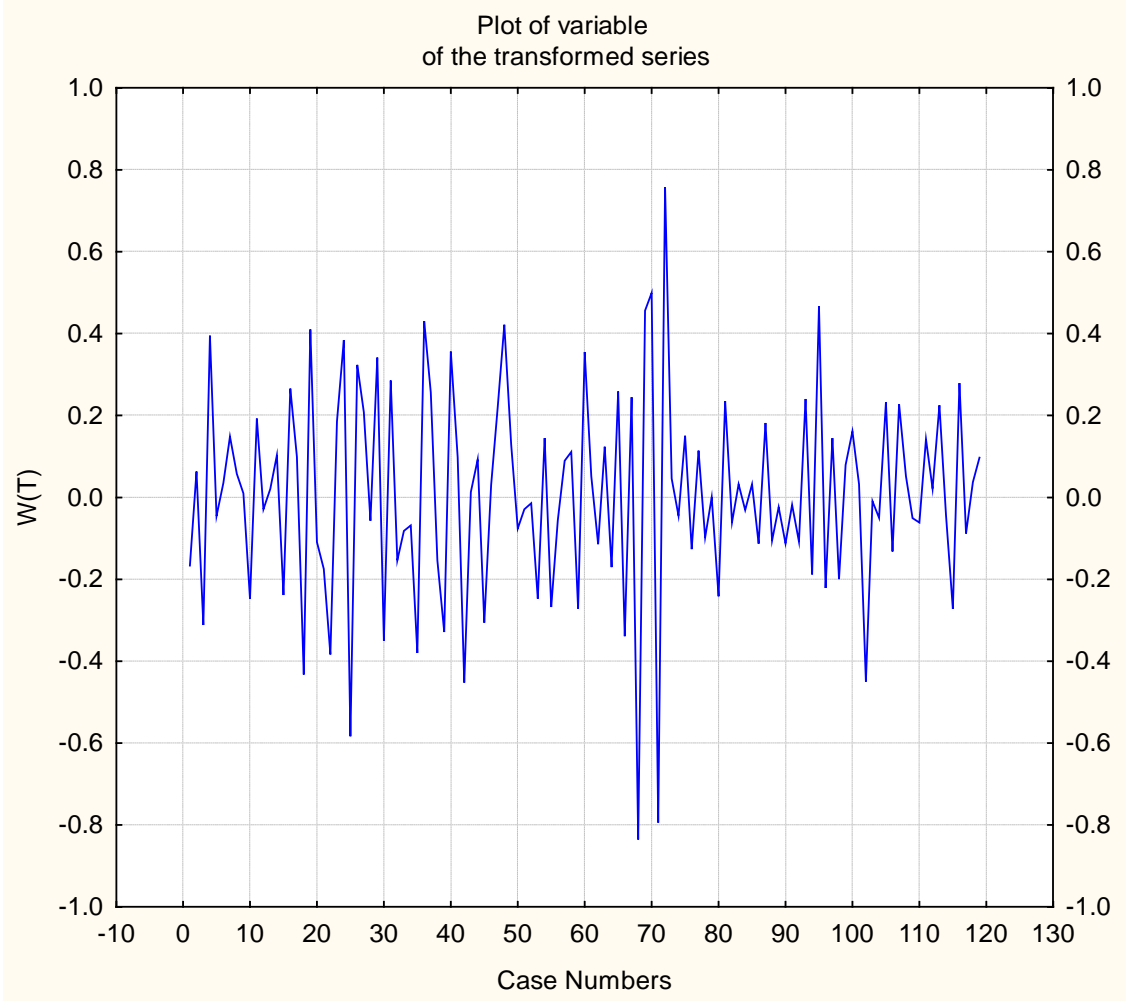

(a) 


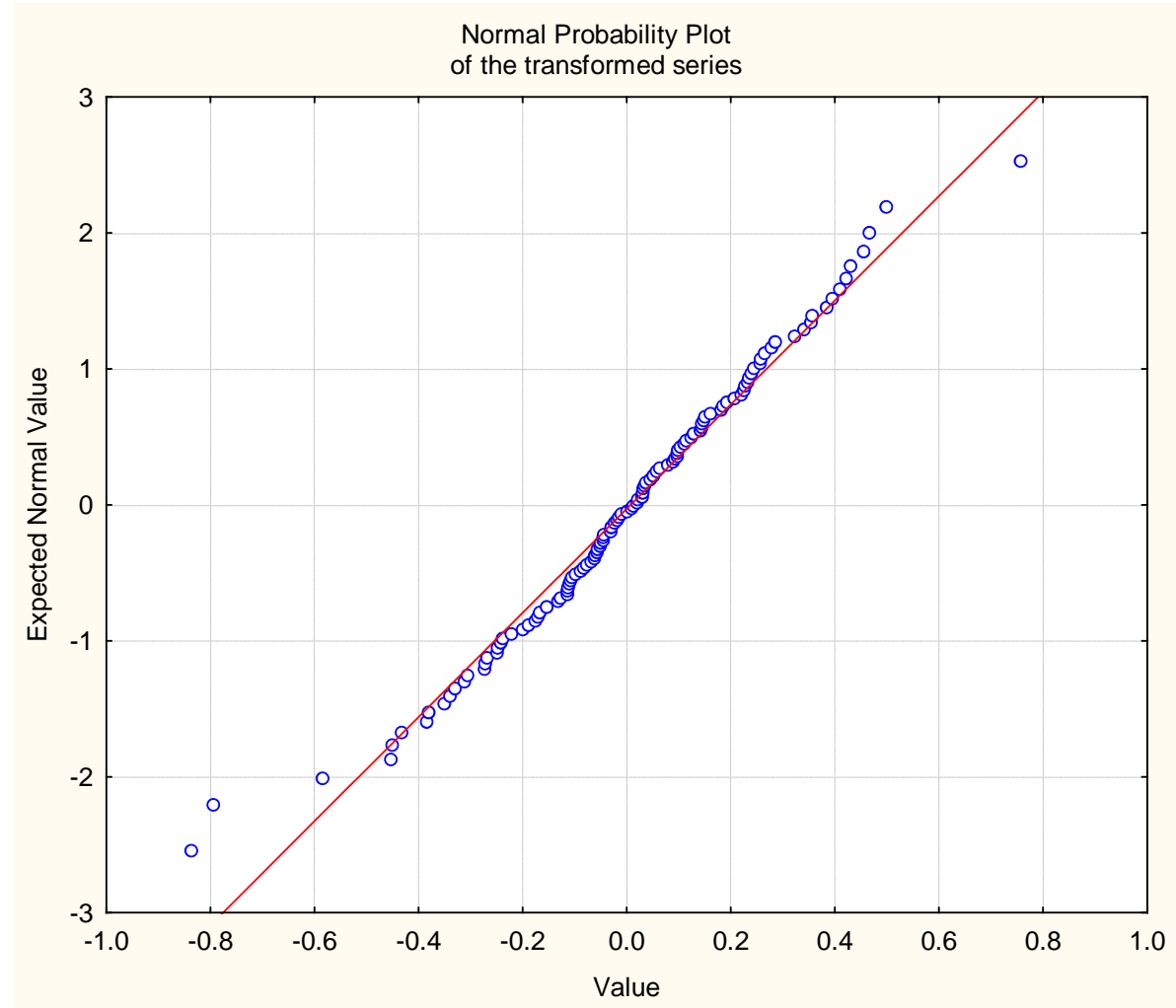

(b)

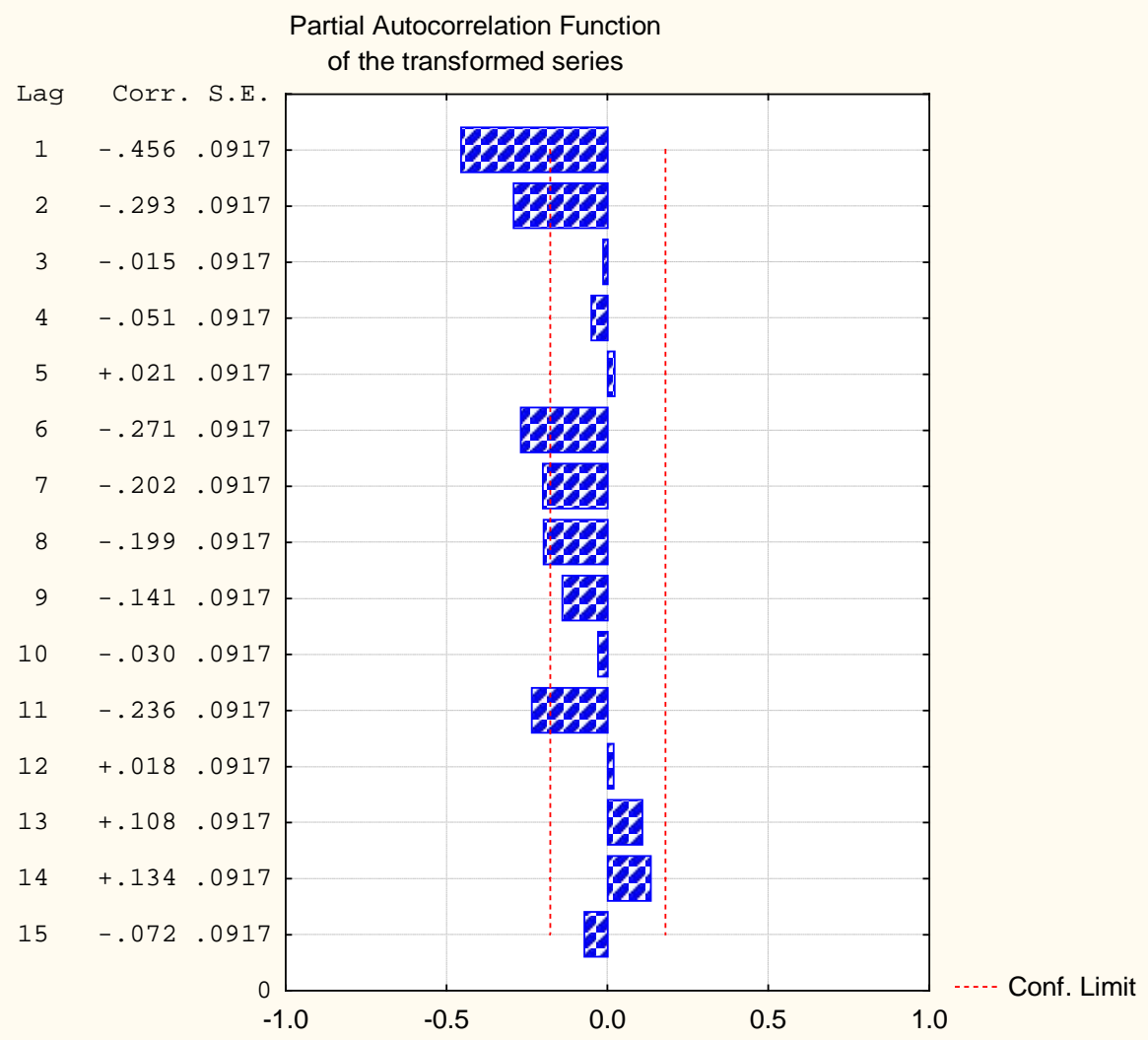

(c) 


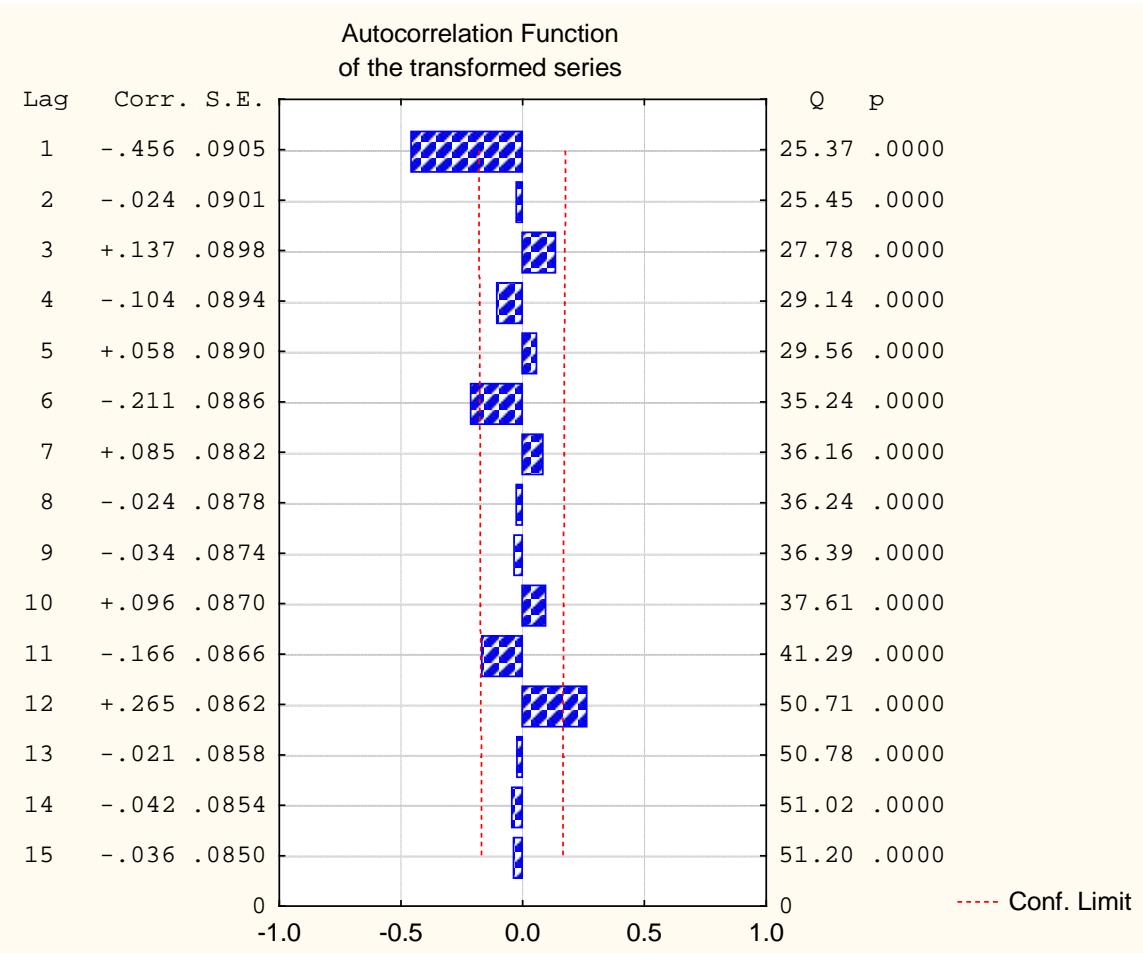

(d)

Figure 2. Graph, normal drawing, self-linking function, and partial self-link function respectively for the converted series.

possibility of using nonlinear mathematical models, so we tried to construct self-regression models from the first rank nonlinear multiplexers and exponential models and different degrees, statistica 8.0.550 for the above models.

The following table represents the models obtained.

(First-order exponential regression models).

\begin{tabular}{ccc}
\hline Statistic Model & Residual variance & NBIC \\
\hline $\operatorname{EXPAR}(1) ; i=1$ & 0.054635 & -2.826215 \\
$\operatorname{EXPAR}(1) ; i=2$ & 0.069568 & -2.584587 \\
$\operatorname{EXPAR}(1) ; i=3$ & 0.054629 & -2.826326 \\
$\operatorname{EXPAR}(1) ; i=4$ & 0.069567 & -2.584595 \\
$\operatorname{EXPAR}(1) ; i=5$ & 0.054628 & -2.826335 \\
$\operatorname{EXPAR}(1) ; i=6$ & 0.069567 & -2.584595 \\
$\operatorname{EXPAR}(1) ; i=7$ & 0.069567 & -2.584595 \\
$\operatorname{EXPAR}(1) ; i=8$ & 0.069567 & -2.584595 \\
\hline
\end{tabular}

14-Studying the stability of the non-linear exponential self-regression model of grade 5 and the first order using the rounding method:

The following model was obtained

$$
X_{t}=\left(-0.973027+0.151226 \mathrm{e}^{-x_{t-1}^{2}}\right)^{5} X_{t-1}+Z_{t}
$$


By applying the theoretical aspect of the previous paragraph, the following results were obtained:

- Single point:

$$
\xi= \pm \sqrt{-\ln \left(\frac{1-\varnothing_{1}}{\pi_{1}}\right)}= \pm \sqrt{-\ln \left(\frac{1-(-0.973027)}{0.151226}\right)}= \pm 3.353648
$$

- Single point stability:

Using equation $|A|<1$ and using similar mathematical operations we obtained

$$
\begin{gathered}
\xi_{t}=\xi_{t-1}\left(\varnothing_{1}^{5}+5 \varnothing_{1}^{4} \pi_{1} \mathrm{e}^{\ln \left(\frac{1-\varnothing_{1}}{\pi_{1}}\right)}+10 \varnothing_{1}^{3} \pi_{1}^{2} \mathrm{e}^{2 \ln \left(\frac{1-\varnothing_{1}}{\pi_{1}}\right)}+10 \varnothing_{1}^{2} \pi_{1}^{3} \mathrm{e}^{3 \ln \left(\frac{1-\varnothing_{1}}{\pi_{1}}\right)}\right. \\
+5 \varnothing_{1} \pi_{1}^{4} \mathrm{e}^{4 \ln \left(\frac{1-\varnothing_{1}}{\pi_{1}}\right)}+\pi_{1}^{5} \mathrm{e}^{5 \ln \left(\frac{1-\varnothing_{1}}{\pi_{1}}\right)}-20 \varnothing_{1} \pi_{1} \mathrm{e}^{\ln \left(\frac{1-\varnothing_{1}}{\pi_{1}}\right)} \\
-60 \varnothing_{1}^{2} \pi_{1}^{2} \mathrm{e}^{2 \ln \left(\frac{1-\varnothing_{1}}{\pi_{1}}\right)}-100 \varnothing_{1}^{3} \pi_{1}^{3} \mathrm{e}^{3 \ln \left(\frac{1-\varnothing_{1}}{\pi_{1}}\right)} \\
\left.-60 \varnothing_{1}^{4} \pi_{1}^{4} \mathrm{e}^{4 \ln \left(\frac{1-\varnothing_{1}}{\pi_{1}}\right)}-20 \varnothing_{1}^{5} \pi_{1}^{5} \mathrm{e}^{5 \ln \left(\frac{1-\varnothing_{1}}{\pi_{1}}\right)}\right) \\
\varnothing_{1}=-0.973027 \\
\pi_{1}=0.151226 \\
\xi_{t}=B \xi_{t-1}
\end{gathered}
$$

where

$$
\begin{aligned}
B= & \varnothing_{1}^{5}+5 \varnothing_{1}^{4} \pi_{1} \mathrm{e}^{\ln \left(\frac{1-\varnothing_{1}}{\pi_{1}}\right)}+10 \varnothing_{1}^{3} \pi_{1}^{2} \mathrm{e}^{2 \ln \left(\frac{1-\varnothing_{1}}{\pi_{1}}\right)}+10 \varnothing_{1}^{2} \pi_{1}^{3} \mathrm{e}^{3 \ln \left(\frac{1-\varnothing_{1}}{\pi_{1}}\right)} \\
& +5 \varnothing_{1} \pi_{1}^{4} \mathrm{e}^{4 \ln \left(\frac{1-\varnothing_{1}}{\pi_{1}}\right)}+\pi_{1}^{5} \mathrm{e}^{5 \ln \left(\frac{1-\varnothing_{1}}{\pi_{1}}\right)}-20 \varnothing_{1} \pi_{1} \mathrm{e}^{\ln \left(\frac{1-\varnothing_{1}}{\pi_{1}}\right)} \\
& -60 \varnothing_{1}^{2} \pi_{1}^{2} \mathrm{e}^{2 \ln \left(\frac{1-\varnothing_{1}}{\pi_{1}}\right)}-100 \varnothing_{1}^{3} \pi_{1}^{3} \mathrm{e}^{3 \ln \left(\frac{1-\varnothing_{1}}{\pi_{1}}\right)} \\
& -60 \varnothing_{1}^{4} \pi_{1}^{4} \mathrm{e}^{4 \ln \left(\frac{1-\varnothing_{1}}{\pi_{1}}\right)}-20 \varnothing_{1}^{5} \pi_{1}^{5} \mathrm{e}^{5 \ln \left(\frac{1-\varnothing_{1}}{\pi_{1}}\right)}
\end{aligned}
$$

Equation (18) represents a first-order differential equation and the characteristic equation is:

$$
\lambda-B=0
$$

When compensating for the values of $\pi_{1}, \varnothing_{1}$ in the previous equation we obtain

$$
B=-0.035189
$$

That is, the root of the equation, and that the condition of the stability is clear that the above model has a single point where stable.

- Stability of the end cycle:

The proposed model is

$$
X_{t}=\left(\varnothing_{1}+\pi_{1} \mathrm{e}^{-x_{t-1}^{2}}\right)^{5} X_{t-1}+Z_{t}
$$


After compensation for $X_{t}, \quad X_{t-1}$ we get the

$$
\xi_{t+q}=\prod_{i=1}^{q}\left(\sum_{j=0}^{5}\left(\begin{array}{c}
5 \\
j
\end{array}\right) \varnothing_{1}^{5-j}\left(\pi_{1} \mathrm{e}^{-\left(X_{t+q-i}\right)^{2}}\right)^{j}\left(1-2 j\left(X_{t+q-i}\right)^{2}\right)\right) \xi_{t}
$$

As a stable condition the end cycle is to achieve the condition $\left|\frac{\xi_{t+q}}{\xi_{t}}\right|<1 \quad$ (note the theory).

Taking point $X_{0}=2.5$ is close to the single point we get

$$
\left|\frac{\xi_{t+q}}{\xi_{t}}\right|=|3.014656|>1
$$

The stability condition of the end cycle is to be $\left|\frac{\xi_{t+q}}{\xi_{t}}\right|<1$ and it is clear that the model above has an unstable end cycle. This confirms what was discussed in the theoretical aspect that if the model has a single point of stability it has an unstable end cycle.

\section{Conclusions and Recommendations}

Through our study of the exponential self-regression model of the first order and the degree $p$, it was shown that the model has non-zero:

$$
\xi= \pm \sqrt{-\ln \left(\frac{1-\varnothing_{1}}{\pi_{1}}\right)}
$$

It has an unstable end cycle.

We recommend that similar models should be studied with higher grades and higher grades.

\section{References}

[1] Naciri, O.S. (2008) Studying the Conditionality of the Cochian Model of the Smooth Transition of Self-Regression with Application. Master Thesis, Faculty of Education, University of Tikrit, Tikrit.

[2] Hamdani, R.A. (2007) Studying the Stability of Some Time Series Models with Their Applications. Master Thesis, Faculty of Education, University of Tikrit, Tikrit.

[3] Ozaki, T. (1982) The Statistical Analysis of Perturbed Limit Cycle Processes Using Nonlinear. Journal of Time Series Analysis, 3, 29-41. https://doi.org/10.1111/j.1467-9892.1982.tb00328.x

[4] Priestley, M.B. (1982) Spectral Analysis and Time Series. Academic Press Inc., London.

[5] Box, C.E.P. and Cox, D.R. (1964) An Analysis of Transformation. J.R. stat. soc. B, No. 26, 211-233.

[6] Chen, S. and Billings, S.A. (1989) Modlling and Analysis of Non-Linear Time Series. University of Southampton, Southampton.

[7] Rahman, A.M.A. (2002) Methods of Statistical Prediction C1. King Saud University, Riyadh. 